\title{
An alternative Candida spp. cell wall disruption method using a basic sorbitol lysis
} buffer and glass beads

\begin{abstract}
This report describes a modified, cost-effective method of cell wall disruption for the yeast Candida spp., which employs the use of glass beads in a simple sorbitol lysis buffer. This method can be used in conjunction with a commercial RNA or genomic DNA isolation method to obtain high-quality RNA or DNA.
\end{abstract}

Keyword: Yeast; Cell wall disruption; Sorbitol lysis buffer; Glass beads; Total RNA isolation; Genomic DNA isolation; Candida 